\title{
Splitting an expander graph
}

\author{
Alan M. Frieze $\quad$ Michael Molloy ${ }^{*}$
}

\begin{abstract}
Let $G=(V, E)$ be an $r$-regular expander graph. Certain algorithms for finding edge disjoint paths require the edges of $G$ to be partitioned into $E=E_{1} \cup E_{2} \cup \cdots \cup E_{k}$ so that the graphs $G_{i}=\left(V, E_{i}\right)$ are each expanders. In this paper we give a non-constructive proof of a very good split plus an algorithm which improves on that given in Broder, Frieze and Upfal, Existence and construction of edge disjoint paths on expander graphs, SIAM Journal on Computing 23 (1994) 976-989.
\end{abstract}

\section{Introduction}

Let $G=(V, E)$ be an $r$-regular graph with $|V|=n$. For the asymptotics we shall assume that $r$ is fixed as $n \rightarrow \infty$. For $S \subseteq V$ let $\operatorname{out}(S)=\{e=(v, w) \in$ $V: ; v \in S, w \notin S\}$ be the set of edges of $G$ with exactly one endpoint in $S$. Let $\left.\Phi_{S}=\mid \operatorname{out}(S)\right) /|S|$ and let the (edge)-expansion $\Phi=\Phi(G)$ of $G$ be defined by

$$
\Phi=\min _{\substack{S \subseteq V \\|S| \leq n / 2}} \Phi_{S}
$$

Loosely speaking, $G$ is an expander if $\Phi$ is "large".

There have been several papers recently ([4], [5], [7], [8], [9]) which deal with the problem of joining selected pairs of vertices by edge-disjoint paths. In all of these papers we are given an expander graph and there is a need to partition the edges $E=E_{1} \cup E_{2} \cup \cdots \cup E_{k}$ so that the graphs $G_{i}=$ $\left(V, E_{i}\right), 1 \leq i \leq k$ are expanders. A method was described in [4], but it is relatively inefficient. This computational problem seems intersting in its own right. In this paper we prove two results. One is non-constructive and

\footnotetext{
*Department of Mathematical Sciences, Carnegie-Mellon University,Pittsburgh PA 15213, USA. Supported in part by NSF grant CCR9530974. E-mail: alan@random.math.cmu.edu

${ }^{\dagger}$ Department of Computer Science,University of Toronto,Toronto, Canada. Supported in part by NSERC grant ????. E-mail: molloy@cs.toronto.edu
} 
shows what might be achieved. The second is constructive. The split is not as good, but it does improve significantly on what is achieved in [4].

We use a subscript $i$ to denote graph-theoretic constructs related to $G_{i}$. Thus $d_{i}(v)$ is the degree of $v$ in $G_{i}$. Left unsubscripted, such things refer to $G$. Thus $d(v)=r$.

In Section 2 we prove

Theorem 1 Let $k \geq 2$ be a positive integer and let $\epsilon>0$ be a small positive real number. Suppose that

$$
\begin{aligned}
\frac{r}{\log r} & \geq 7 k \epsilon^{-2} \\
\Phi & \geq 4 \epsilon^{-2} k \log r .
\end{aligned}
$$

Then there exists a partition $E=E_{1} \cup E_{2} \cup \cdots \cup E_{k}$ such that for $1 \leq i \leq k$

(a)

$$
\Phi_{i} \geq(1-\epsilon) \frac{\Phi}{k}
$$

(b)

$$
(1-\epsilon) \frac{r}{k} \leq \delta\left(G_{i}\right) \leq \Delta\left(G_{i}\right) \leq(1+\epsilon) \frac{r}{k} .
$$

We have not been able to make the proof of this theorem constructive as in [3], [1] and [10]. Instead we will suffice ourselves with the following theorem, proved in Section 3. Assume that

$$
\Phi_{S} \geq(1-\alpha) r|S|
$$

for $|S| \leq \gamma n$. For random $r$-regular graphs and Ramanujan graphs we can take $\gamma$ to be a small constant and $\alpha=O\left(\gamma+\frac{1}{\sqrt{r}}\right)$.

Theorem 2 Assume that (1) holds. There is a randomised polynomial time algorithm $\left(O\left(n^{O(\ln r)} \log \delta^{-1}\right)\right)$ which with probability at least $1-\delta$ constructs $E_{1}, E_{2}, \ldots, E_{k}$ such that

$$
\Phi_{i} \geq(1-\epsilon) \frac{\Phi}{k}-\left(\frac{\alpha}{2}+\epsilon\right) r
$$

for $i=1,2, \ldots, k$. 
This theorem is only useful if $\Phi \geq c r$ for some $c$ satisfying $c \gg \alpha$. Nevertheless, its requirements are weaker than those needed in [4] and the conclusion is stronger. Notice that our two examples, $r$-regular graphs and Ramanujan graphs satisfy the required condition. In the context of finding edge disjoint paths, it is enough that $\Phi_{i}>1$ for $i=1,2, \ldots, k$.

Note that there is not time to verify that the algorithm succeeds. Instead, in the applications, we assume it has and repeat the split if the algorithm that uses fails to find the required paths.

\section{Existence Result}

In this section, we prove Theorem 1 . We will use the general version of the Lovász Local Lemma. For each $e \in E$ we randomly choose an integer $i \in[k]$ and then place $e$ in $E_{i}$. We must show that there is a positive probability of choosing a partition which satisfies the conditions of the theorem.

We define the following sets of bad events: If $S \subseteq V$ then $G[V]=\left(S, E_{S}\right)$ is the subgraph of $G$ induced by $T$. Thus $E_{S}=\{e \in T: e \subseteq S\}$.

(a) For $v \in V$ and $i \in[k]$,

$$
A_{v, i}=A_{\{v\}, i}=\left\{d_{i}(v) \notin[(1-\epsilon) r / k,(1+\epsilon) r / k]\right\} .
$$

(b) For $S \subseteq V, 2 \leq|S| \leq n / 2, G[S]$ connected and $i \in[k]$,

$$
A_{S, i}=\left\{\left|\operatorname{out}_{i}(S)\right|<(1-\epsilon)|\operatorname{out}(S)| / k\right\} .
$$

In showing that $\Phi_{i}$ is sufficiently large we can restrict our attention to out $(S)$ for which $G[S]$ is connected. Indeed, for $S \subset V$ let $C_{1}, C_{2}, \ldots, C_{t}$ be the components of $G[S]$. Then

$$
\Phi_{S} \geq \min _{s=1}^{t} \frac{\left|\operatorname{out}_{i}\left(C_{s}\right)\right|}{\left|C_{s}\right|}
$$

We now consider the dependency graph of the bad events.

Claim 1 For $v \in V$ there are at most $(e r)^{s-1}$ sets $S$ such that (i) $v \in S$, (ii) $|S|=s$ and (iii) $G[S]$ is connected.

Proof of Claim 1 The number of such sets is bounded by the number of distinct $s$-vertex trees which are rooted at $v$. This in turn is bounded by the number of distinct $r$-ary rooted trees with $s$ vertices. This is equal to $\left(\begin{array}{c}r s \\ s\end{array}\right) /((r-1) s+1)$, see Knuth [6]. 


\section{End of proof of Claim 1}

The Chernoff bounds for the tails of the binomial distribution $B(n, p)$ that we use are

$$
\begin{aligned}
& \operatorname{Pr}(B(n, p) \geq(1+\epsilon) n p) \leq e^{-\epsilon^{2} n p / 3} \\
& \operatorname{Pr}(B(n, p) \leq(1+\epsilon) n p) \leq e^{-\epsilon^{2} n p / 2}
\end{aligned}
$$

where $0 \leq \epsilon \leq 1$.

Using them we obtain,

$$
\begin{aligned}
\operatorname{Pr}\left(A_{v, i}\right) & \leq 2 e^{-\epsilon^{2} r /(3 k)} \\
& \leq 2 e^{-(7 \log r) / 3} \\
& <\frac{1}{r^{2}} . \\
\operatorname{Pr}\left(A_{S, i}\right) & \leq \exp \left\{-\frac{\epsilon^{2}|\operatorname{out}(S)|}{2 k}\right\} \\
& \leq e^{-2|S| \log r} \\
& =\frac{1}{r^{2|S|}} .
\end{aligned}
$$

Now, for $S \subseteq V, 1 \leq|S| \leq n / 2$ and $G[S]$ connected, let

$$
x_{S, i}=\left(\frac{2}{r^{2}}\right)^{|S|} .
$$

We show that

$$
\operatorname{Pr}\left(A_{S, i}\right)<x_{S, i} \prod_{(S, i) \sim(T, j)}\left(1-x_{T, j}\right),
$$

where $(S, i) \sim(T, j)$ denotes adjacency of $A_{S, i}$ and $A_{T, j}$ in the dependency graph of bad events i.e. out $(S) \cap \operatorname{out}(T) \neq \emptyset$. The theorem then follows from the general version of the local lemma, see for example Alon and Spencer $[2]$.

It follows from Claim 1 that if $|S|=s$ then there are at most $k s(e r)^{t}$ events $A_{T, j}$ with $|T|=t$ such that $(S, i) \sim(T, j)$. Thus, using $1-x \geq e^{-2 x}$ for 
$0 \leq x \leq 1 / 2$ we have

$$
\begin{aligned}
x_{S, i} \prod_{(S, i) \sim(T, j)}\left(1-x_{T, j}\right) & \geq\left(\frac{2}{r^{2}}\right)^{s} \prod_{t \geq 1}\left(1-\left(\frac{2}{r^{2}}\right)^{t}\right)^{k s(e r)^{t}} \\
& \geq\left(\frac{2}{r^{2}}\right)^{s} \exp \left\{-2 k s \sum_{t \geq 1}\left(\frac{2 e}{r}\right)^{t}\right\} \\
& =\left(\frac{2}{r^{2}}\right)^{s} \exp \left\{-\frac{4 k e s}{r-2 e}\right\} \\
& >\frac{1}{r^{2 s}},
\end{aligned}
$$

provided

$$
r>2 e+\frac{4 k e}{\ln 2} .
$$

In which case (4) holds, proving the theorem.

\section{Splitting Algorithm}

In this section, we prove Theorem 2 .

Idea: We will define a sequence of sets $V=B_{1} \supseteq B_{2} \supseteq \cdots \supseteq B_{t}$ such that if $S \subseteq B_{j} \backslash B_{j+1}$ then $\operatorname{out}_{i}(S)$ is large enough and further that every vertex in $B_{j} \backslash B_{j+1}$ has few neighbours in $B_{j+1}$. Then we will see that this latter condition accounts for the $-\left(\frac{\alpha}{2}+\epsilon\right) r$ term in the theorem.

Assume we have $B \subseteq V$. Initially, $B=V$. We randomly colour the edges of $G$ which are incident with $B$, with $k$ colours. Note that

$$
\begin{aligned}
\operatorname{Pr}\left(\exists S \subseteq B, i \in[k] \text { s.t. }|S|>2 \log _{2} n, G[S] \text { is connected and } \Phi_{i, S} \leq\left(\frac{1-\epsilon}{k}\right) \Phi\right) & \\
& \leq k n \sum_{t \geq 2 \log _{2} n}(e r)^{t-1} e^{-\epsilon^{2} \Phi t /(2 k)} \\
& \leq 2 k n(e r)^{2 \log _{2} n} e^{-\epsilon^{2} \Phi 2 \log _{2} n /(2 k)} \\
& \leq \frac{2 k}{n} .
\end{aligned}
$$

So, in a sense the large sets, take care of themselves. Now consider the smaller sets. Let

$$
\begin{aligned}
X_{0}=\left\{v: \exists S \subseteq B,|S| \leq 2 \log _{2} n, G[S] \text { is connected, } v \in S\right. \\
\text { and } \left.i \in[k] \text { s.t. } \Phi_{i, S} \leq\left(\frac{1-\epsilon}{k}\right) \Phi\right\} .
\end{aligned}
$$


$X_{0}$ can be constructed in $O\left(n(e r)^{2 \log _{2} n}\right)=O\left(n^{O(\ln r)}\right)$ time.

$$
\begin{aligned}
\mathbf{E}\left(\left|X_{0}\right|\right) & \leq|B| \sum_{t=1}^{2 \log _{2} n}(e r)^{t-1} e^{-\epsilon^{2} \Phi t /(2 k)} \\
& \leq 2|B| e^{-\epsilon^{2} \Phi /(2 k)}
\end{aligned}
$$

provided

$$
\Phi \geq \frac{2 k}{\epsilon^{2}} \ln (2 e r)
$$

In which case

$$
\mathbf{E}\left(\left|X_{0}\right|\right) \leq \frac{|B|}{e r}
$$

Assume now that

$$
r \geq \frac{1}{e \gamma}\left(\frac{\alpha}{\epsilon}+1\right)
$$

Then

$$
\operatorname{Pr}\left(\left|X_{0}\right| \geq \frac{2|B|}{e r}\right) \leq \frac{1}{2}
$$

We repeat the above colouring until we find that $\left|X_{0}\right| \leq \frac{2|B|}{e r}$. Now define $X_{j}=X_{0} \cup\left\{v_{1}, v_{2}, \ldots, v_{j}\right\}$ where $v_{j}$ has at least $\left(\frac{\alpha}{2}+\epsilon\right) r$ neighbours in $X_{j-1}$. Now $|S| \leq \gamma n$ implies that $S$ contains at most

$$
\frac{1}{2}(r|S|-|\operatorname{out}(S)|) \leq \frac{1}{2} \alpha r|S|
$$

edges. Furthermore, $X_{j}$ has at least $\left(\frac{\alpha}{2}+\epsilon\right) r j$ edges and at most $j+\frac{2|B|}{e r}$ vertices. Thus this process stops before $j$ reaches $\frac{\alpha|B|}{\epsilon e r}$. So if $X$ denotes $X_{j}$ when $v_{j+1}$ cannot be found, then

$$
|X| \leq \gamma|B|
$$

We will repeat the construction with $B$ replaced by $X$. Let $V=B_{1} \supseteq B_{2} \supseteq$ $\cdots \supseteq B_{t}$ be the sequence of sets constructed. $B_{t}$ will be the first set of size at most $\ln n$. We can "brute force" colour the edges incident with $B_{t}$ so that every subset $S$ of $B_{t}$ satisfies $\Phi_{i, S} \geq\left(\frac{1-\epsilon}{k}\right) \Phi$. We use Theorem 1 to justify the success of this. The sequence of sets $B_{1}, B_{2}, \ldots, B_{t}$ satisfies

- $\left|B_{j}\right| \leq \gamma^{j} n$.

- $S \subseteq B_{j} \backslash B_{j+1}$ implies $\Phi_{i, S} \geq\left(\frac{1-\epsilon}{k}\right) \Phi$.

- $v \in B_{j} \backslash B_{j+1}$ implies $v$ has at most $\left(\frac{\alpha}{2}+\epsilon\right) r j$ neighbours in $B_{j+1}$. 
So if $S \subseteq V$ and $S_{j}=S \cap\left(B_{j} \backslash B_{j+1}\right)$ then

$$
\begin{aligned}
\left|\operatorname{out}_{i}(S)\right| & \geq \sum_{j=1}^{t-1}\left(\left|\operatorname{out}_{i}\left(S_{j}\right)\right|-\left|e\left(S_{j}: S_{j+1}\right)\right|\right)+\left|\operatorname{out}_{i}\left(S_{t}\right)\right| \\
& \geq \sum_{j=1}^{t-1}\left(\frac{1-\epsilon}{k} \Phi-\left(\frac{\alpha}{2}+\epsilon\right) r\right)\left|S_{j}\right|+\left(\frac{1-\epsilon}{k}\right) \Phi\left|S_{t}\right| \\
& \geq\left(\frac{1-\epsilon}{k} \Phi-\left(\frac{\alpha}{2}+\epsilon\right) r\right)|S|
\end{aligned}
$$

\section{References}

[1] N.Alon, A parallel algorithmic version of the Local Lemma, Random Structures and Algorithms 2 (1991) 366-378.

[2] N.Alon and J.H.Spencer, The Probabilistic Method, Wiley, 1992.

[3] J.Beck, An algorithmic approach to the Lovász local lemma, Random Structures and Algorithms 2 (1991) 343-365.

[4] A.Z.Broder, A.M.Frieze and E.Upfal, Existence and construction of edge-disjoint paths on expander graphs, SIAM Journal of Computing 23 (1994) 976-989.

[5] A.Z.Broder, A.M.Frieze and E.Upfal, Existence and construction of edge low congestion paths on expander graphs, Proceedings of the 29th Annual ACM Symposium on Theory of Computing, (1997) 531-539.

[6] D.E.Knuth, The art of computer programming, Volume 1, Fundamental Algorithms, Addison-Wesley, 1968.

[7] T.Leighton and S.Rao, Circuit switching: a multicommodity flow based approach, Proceedings of a Workshop on Randomized Parallel Computing 1996.

[8] T.Leighton, S.Rao and A.Srinivasan, Multi-commodity flow and circuit switching, Proceedings of the Hawaii International Conference on System Sciences, 1998.

[9] T.Leighton, S.Rao and A.Srinivasan, New algorithmic aspects of the local lemma with applications to partitioning and routing, to appear in Proceedings of SODA '99. 
[10] M.Molloy and B.Reed, Further algorithmic aspects of the local lemma, Proceedings of the 30th Annual ACM Symposium on Theory of Computing (1998) 524-529. 